\title{
PLEIADES-HR SYSTEM PRODUCTS PERFORMANCE AFTER IN-ORBIT COMMISSIONING PHASE
}

\author{
C. Panem, F. Bignalet-Cazalet, S. Baillarin \\ CNES, 18 Av. Edouard Belin, 31401 Toulouse Cedex 9, France \\ (chantal.panem, françois.bignalet-cazalet, simon.baillarin)@ cnes.fr
}

Special Session ISPRS and IAA: Pléiades System and Acquisition Capability

KEY WORDS: optical mission, high resolution, agility, image processing

\begin{abstract}
:
Launched on 2011, December 16th by the second Soyuz operated in French Guiana, PLEIADES-HR acquired its first high resolution images 3 days after. The PLEIADES program is a space Earth Observation system led by the French Space Agency (CNES), it provides the first european high resolution satellite which will simultaneously acquire in Panchromatic and MultiSpectral modes $20 \mathrm{~km}$ wide images with a $70 \mathrm{~cm}$ nadir resolution.

Imaging capabilities have been highly optimized in order to acquire, in the same pass, along-track mosaics, stereo pairs and triplets, and multi-targets. Ground segment processes automatically data to ensure operational requirements and quick access to images for the users. Since ground processing capabilities have been taken into account very early in the mission development, it has been possible to relax some costly on-board components requirements, in order to achieve a cost effective on-board/ground compromise. Starting from the PLEIADES system and on board characteristics, this paper first presents an overview of ground segment functional breakdown. Then it focuses more precisely on the different levels of products and associated processing performances. Finally the paper shows how appropriate ground processing systems allowed CNES Image Quality team to assess radiometric and geometric performances during the 6 first months of PLEIADES 1A.
\end{abstract}

\section{INTRODUCTION TO PLEIADES-HR SYSTEM}

Monitoring human activities and earth resources needs to access to more and more detailed information. In this frame, PLEIADES system will consist of 2 optical satellites with an improved submetric resolution of $0.7 \mathrm{~m}$ compared to the 2.5 meters of SPOT 5 images.

Moreover, even though Pleiades swath only covers $20 \mathrm{~km}$ at nadir, the satellites high agility allows to acquire in the same pass a mosaic of images covering a larger area (up to $120 \mathrm{~km} * 120 \mathrm{~km}$ ), or stereoscopic images of $300 \mathrm{~km}$ long.

The constellation of 2 identical satellites also provides veryhigh-resolution data products in short time and offers a daily revisit to any point on the globe with an acquisition capability of up to 450 images per day and per satellite (considering $20 \times 20 \mathrm{~km}^{2}$ images).

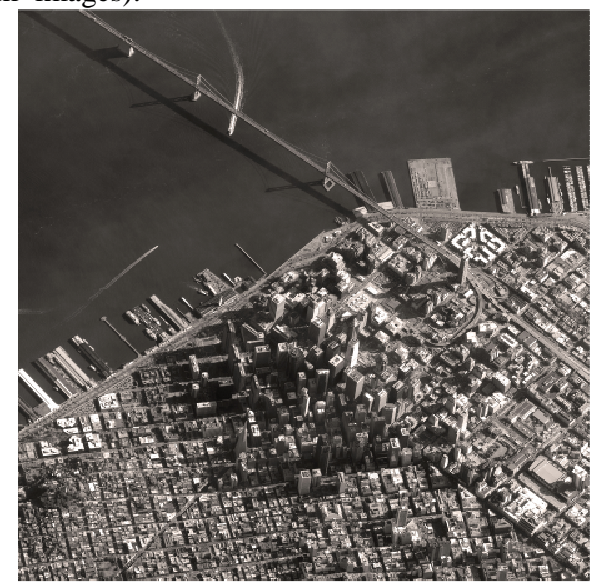

Figure 1 : Pleiades-HR image of San Francisco, (C) CNES Copyright 2012
The first PLEIADES satellite was launched from Kourou (French Guiana) 16 ${ }^{\text {th }}$ December 2012 and has successfully passed the In-Orbit Commissioning Phase (Lebegue, 2012).

First, this paper offers an overview of the satellite main characteristics and on-board processing chain. Then the ground processing centers are presented, focusing on the image processing chain. Finally, the main system products are detailed.

\section{PLEIADES SATELLITE CHARACTERISTICS}

\subsection{Detectors and focal plane}

Thinned TDI detectors of 6000 pixels are used for panchromatic detection (PAN), with typically 15 integration lines. They can be optimally used thanks to an adequate guidance strategy of the satellite line of sight, including micro-vibrations levels minimization and specific geometrical accommodation of detector lines in the focal plane to minimize optical distortion effects.

The multi-spectral detection (XS) is realized by detectors of 1500 pixels each. Each detector consists of four lines assembly, enabling four colors imaging (blue, green, red, near infrared).

The focal plane is then constituted by two symmetrical arrangements of detectors. To acquire images over a field of view of $20 \mathrm{~km}$, each line of sight is composed by juxtaposing of 5 detectors, generating images of 30000 columns in the PAN channel and 7500 columns in the XS channel.

With a separation mirror, the XS and PAN viewing planes are separated by $1.5 \mathrm{mrad}$ in the field only, which makes PAN and Multispectral channels registration possible by a simple ground processing (re-sampling). 


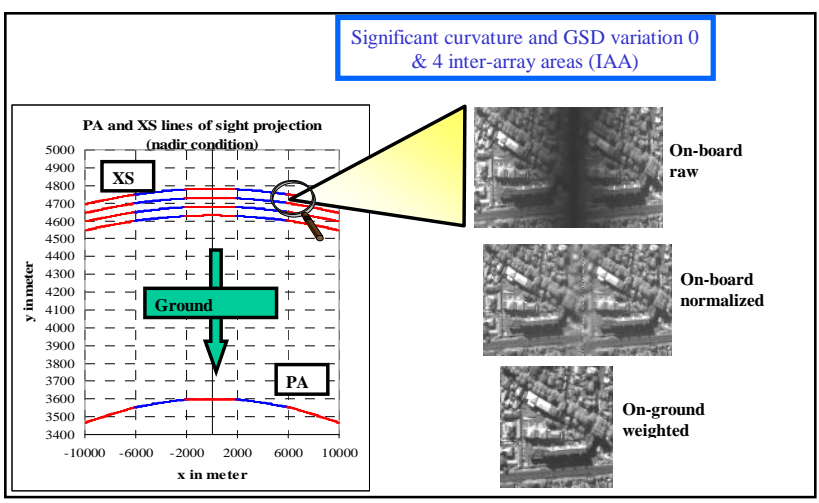

Figure 2: Focal plane and image reconstruction

\subsection{Payload data handling and transmission}

The detectors output is compressed in the Payload Data Compression Unit by sets of 1500 pixels. Compression is performed with a wavelets transform algorithm, that enables the compression ratio to go up to 7 . The video data rate from the instrument is then $4.5 \mathrm{Gbits} / \mathrm{s}$.

The data are multiplexed, ciphered and memorized in the Solid State Mass Memory which has a storage capacity of 600 Gbits. The output rate is of $465 \mathrm{Mbits} / \mathrm{s}$, on three individual channels of $155 \mathrm{Mbits} / \mathrm{s}$ each.

When downlinked data are coded following a trellis-coded scheme in 8-PSK type modulators .

These high storage capacity and high transmission rate allow high reactivity of the Pléiades system with an optimized number of ground image receiving stations. Coupled with an enhanced ground processing, a great amount of user's requests can be satisfied within one day due to high reprogramming capabilities offered by centralized data collection.

\section{GROUND SEGMENT PERFORMANCES}

The overall ground system has been designed to fulfill the operational requirements and to ensure quick access to information in each operational center with an automated production system able to generate $20 \mathrm{~km} \mathrm{x} 20 \mathrm{~km}$ orthorectified images in less than 30 minutes.

Composed of one Secure Dual Control Center (SDGC, main control center for civilian and defense needs), three main Users Ground Centers (UGCs), several Regional Image Receiving station and one Image Calibration Center (ICC), the distributed PLEIADES ground segment is fully operational since In-Orbit acceptance (2012, March, $1^{\text {st }}$ ).

\subsection{Users Centers}

Today 3 civilian users centers (France, Sweden and Japan) and 2 defense centers (France and Spain) are qualified and have the capability to daily program and process the acquired data. 2 more civilian regional stations will be deployed soon.
With 3 mission plans per day and a local direct tasking capacity (Gleyzes, 2012), the system offers a high level of reactivity for images acquisition.

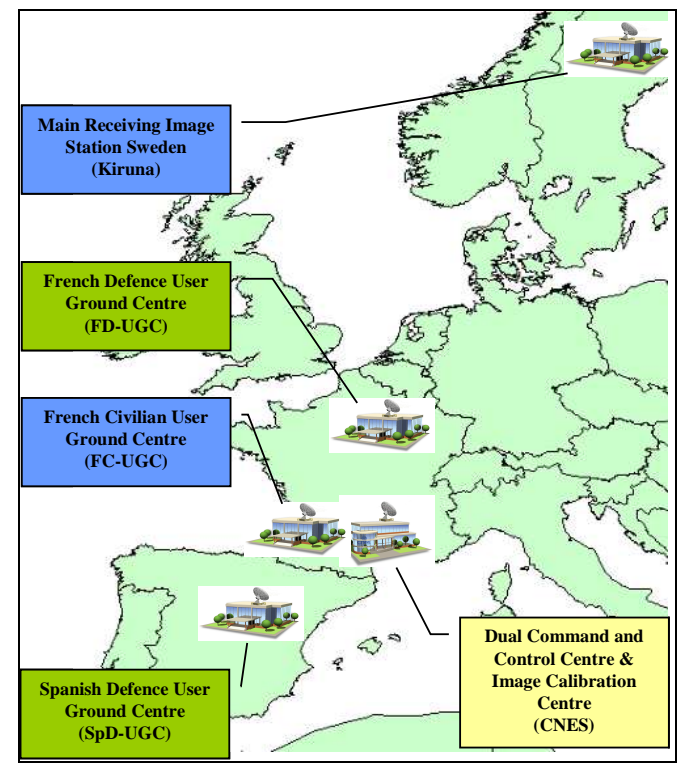

Figure 3: PLEIADES Main centers

According to the scheduled mission plan, the raw telemetry can be down-linked to a given station and then automatically routed to be processed by a local or distant processing center. In less than several minutes for urgent data or one hour for routine ones, images can be displayed by users through the catalog User Access Interface.

Each UGC is in charge of taking into account the image production requests coming from authorized users and of processing the corrections associated to the requested image products.

All users centers have been progressively deployed and then intensively used during the commissioning phase in relation with the ICC in CNES.

The ground processing has been designed to be highly interoperable allowing future federations with other earth observation systems in the GMES context. Hence, data format (catalogued data, final image product) and data access services (online catalogued data browsing, product ordering) definitions have been major issues (Baillarin, 2006).

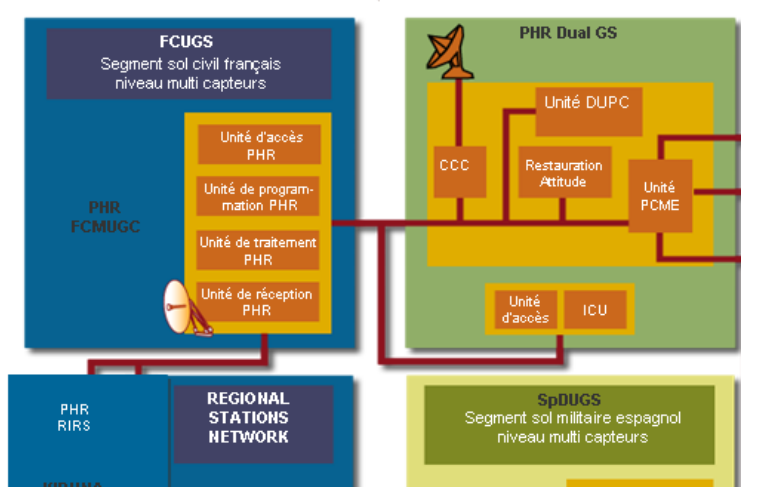

Figure 4: Ground segment architecture 


\subsection{A set of powerful functional units}

Each user center is able to manage the users requests in terms of image acquisition and products generations, they include a set of key components called functional units :

- a X band antenna in charge of satellite acquisition, 3 channels signal demodulation and ingestion system

- an Image Processing Unit (IPU) in charge of inventorying, cataloguing, archiving and producing the images,

- a Programming Unit in charge of managing the mission planning requests.

- a set of Access Units allowing the users to browze the images catalog, submit requests and receive the ordered products.

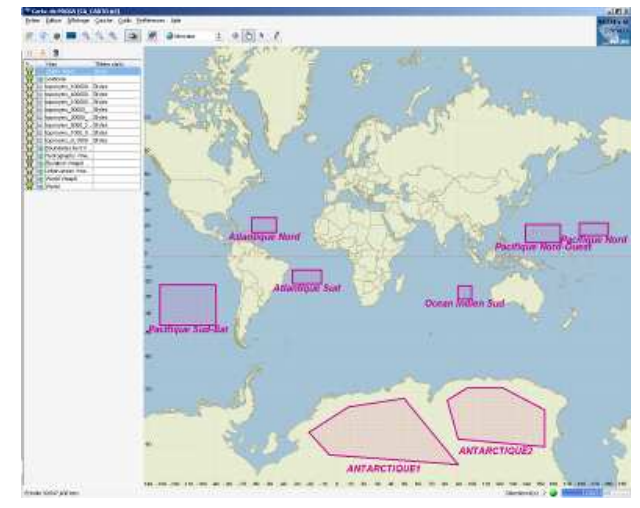

Figure 5: Acquisition zones for ImageQuality Assesment

\subsection{Scalable and automatic Image processing system}

The IPU is then one of the essential components: dealing with huge data volume, high performances and automatisation requirements, this unit is the most consuming one in terms of computer resources in the ground segment. Indeed, as soon as the data are received, it allows the system being fully datadriven. Starting from automatic inventory towards update of the main catalogue, production orders can be automatically or manually generated at the required level so that the products can be quickly delivered to the user. The system is based on a scalable hardware architecture that can be tailored to centers needs, from dozens to hundreds of daily images archiving and products generation.

Urgent data-strips (flagged as it by the mission planning in the telemetry) are automatically sorted by ingestion in the receiving station to be processed first by the Image Processing Unit.
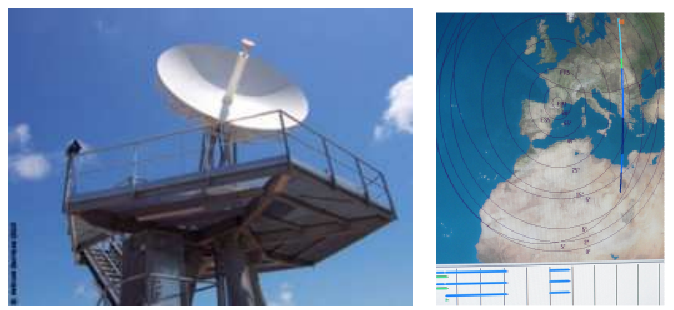

Figure 6 : PLEIADES Receiving station
Automatic inventory of data has to decipher, decompress, analyse telemetry quality and reconstruct images from 40 sets of source data, with auxiliary satellite data processing for album localization and cloud coverage notation.

The performances required for such a system has never been so stringent in the past, in terms of volume of data to process (up to 300 products per day) and image production timeliness taking into account the new complex algorithms like automatic mosaic processing.

Regarding production timeliness, the time between the telemetry download and the availability of the raw product for the customer is less than 1 hour for urgent images, 6 hours for routine ones.

The system is able to process an ortho-image product in less than $15 \mathrm{mn}$ on new hardware generation and a mosaic product $(60 * 60 \mathrm{~km} 2)$ in less than 2 hours.

The Image processing unit is highly scalable, according to the frequency and to the volume of data the center receives. Concerning storage needs, the volume of a Pleiades-HR product goes from 2 GBytes $(20 * 20 \mathrm{~km} 2)$ to 40 GBytes in compressed mode, implying a temporary volume of several TBytes during the product generation process.

The main civilian center has shown its ability to generate 200 products a day. During the first 3 months, 6000 data-strips all over the word have been acquired and archived and 4200 products were delivered to ICC.

\section{SYSTEM PRODUCTS}

\subsection{System products adapted to users needs}

Several types of products have been defined in order to fulfil the different user needs (Kubik, 2005). All of them were very useful during commissioning phase, specially raw levels for calibration activities.

Some users need data superimposed to maps into Geographic Information Systems (GIS), whatever the sensors characteristics. They usually use ortho-image products, resampled into a cartographic projection and corrected from sensor and terrain distortions.

Some users want to access to "raw data" in order to deliver value-added products $(3 \mathrm{D}$, geophysics data, ...) using their own methods. They need comprehensive ancillary data to compute the geometric model. Because of the native geometry of a sensor like Pleiades-HR is very specific, a "Sensor Level" product has been defined making the geometric model simpler while preserving its accuracy.

Hence, two processing levels have been defined in addition to the classical raw levels. They are presented and assessed here after in table 1.

Products are composed of imagery files, quicklook and thumbnail images and metadata files (DIMAP XML file, METAFOR file respecting ISO 19115 format, Ground Processing Parameters) including quality masks. 


\begin{tabular}{|l|l|l|}
\hline $\begin{array}{l}\text { Mission } \\
\text { Require- } \\
\text { ments }\end{array}$ & $\begin{array}{l}\text { Corresponding } \\
\text { Product Level }\end{array}$ & Main characteristics \\
\hline Level 0 & Raw level & Raw data after decompression \\
\hline Level 1A & $\begin{array}{l}\text { Radio- } \\
\text { corrected level }\end{array}$ & $\begin{array}{l}\text { Raw level + radiometric } \\
\text { corrections. PAN restoration is } \\
\text { run optionally. }\end{array}$ \\
\hline Level 1B & $\begin{array}{l}\text { "Perfect } \\
\text { Sensor" level }\end{array}$ & $\begin{array}{l}\text { Corresponds to an image which } \\
\text { would have been acquired by a } \\
\text { perfect Push-Broom sensor. For } \\
\text { that level we use the acronym CP } \\
\text { (for the french 'Capteur Parfait') }\end{array}$ \\
\hline Level 1C & $\begin{array}{l}\text { "Orthoimage" } \\
\text { level }\end{array}$ & $\begin{array}{l}\text { Orthorectified, terrain corrected } \\
\text { Without Ground Control Point } \\
\text { "Mosaic" level }\end{array}$ \\
$\begin{array}{l}\text { Set of orthorectified images in } \\
\text { the same geometry }\end{array}$ \\
\hline
\end{tabular}

Table 1 : Processing levels

Each image file compressed using JPEG2000 (JP2) algorithm, with configuration parameters:

- Tile sizes : 2048x2048,

- $\quad$ Flush period : 2048 lines.

- Codeblock size : 64 (default value)

- Wavelet decomposition level : 5 (default value) (with 4096x4096 tiles, the higher level of decomposition contains $128 \times 128$ coefficients,

- Order : recommanded order is RPCL (Resolution, Position, Color component, Layer quality),

- $\quad$ Markers : ORGGen_plt option is used to allow optimized decompression.

- $\quad$ SPrecision and Qstep are 2 parameters depending on image coding ( 8 or 12 bits). SPrecision $=$ coding dynamic $(8$ or 12) and Qstep $=1 / 2^{\wedge}$ (coding dynamic),

- YCC compression is used for PAN-Sharpened images,

- $\quad$ Rate $=3$ bits by color plane (12 bits for 4 bands, 9 bits for 3 bands, 3 bits for monospectral).

Lossless or lossy compression (nominal mode) algorithms (JPEG2000) are used in order to optimize the products on line delivery.

For image file whose dimensions are larger than a configurable limit ( $>\sim 1$ scene $\sim 2.6$ GBytes), the image file is physically tiled in several JP2 files.

A GML-JP2 header is inserted in each JP2 format, to store geolocation, feature overlay, annotation and text information within JP2 files in Perfect Sensor, ortho and mosaic levels.

\subsection{Archive level}

After inventory process, images are archived at raw level, with binary files kept compressed as on-board source files, auxiliary data, album and mask files. Archive products are internal products that cannot be delivered to end users, but exchanged between user centers and as a basis for any other product generation.

\subsection{Level 0 and level 1 products}

For calibration needs, around 2300 acquisitions over oceans, La Crau test site, deserts, stars and Moon images and also a consequent set of AMETHIST and 'slow motion' images have been acquired and processed by Image Processing Unit, at archive level, L0 or L1 level (Lebegue, 2012).

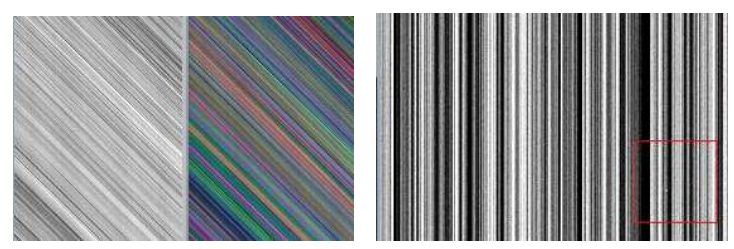

Figure 7 : examples of AMETHIST PAN, XS and slow-Motion images

Level 0 images are raw images composed of 5 adjacent bands. Used by image quality experts for inter-array egalization and signal-to-noise ratio assessment, they allow to update and deliver correction coefficients sets to operational users centres.

Images at level 1 have the same geometry than Level 0 ones but are radiometrically corrected : aberrant detectors correction, inter-array reconstruction, panchromatic restauration.

These products were mainly used for on-board compression rate assessment and optimization. The nominal onboard compression bit rate was optimized on numerous images by qualitative and quantitative analysis and allowed to set the recommended bit rates from 2.50 to 2.86 for PAN band and from 2.86 to 3.33 for XS bands to avoid compression artefacts.

The product is composed of a set of 5 panchromatic decompressed data strips and 5 multispectral 4-band data-strips, one per sub-swath, with auxiliary data file and geometric model file. The volume for a $20 \mathrm{~km} * 20 \mathrm{~km}$ product is around 500 Mbytes with nominal compression.

Processing images at level 0 or level 1 levels requires less than $10 \mathrm{mn}$.

\subsection{Simplifying the focal plane : the "Sensor" Level}

The complexity of the Pleiades-HR focal plane makes the classical level 1 product difficult to use. A new product level called "Sensor Level" is proposed, processing steps has been described in (Baillarin, 2009).

The Sensor Level product is the image that would have been delivered by a standard push-broom sensor (SPOT-like). The product is only corrected from on-board distortions (viewing directions and high frequency attitude variations). All XS and PA pixels are registered.

XS can be PAN-sharpened in the same processing flow to obtain a $0.50 \mathrm{~m}$ GSD 4-band colour image (blue, green, red, near infrared). 


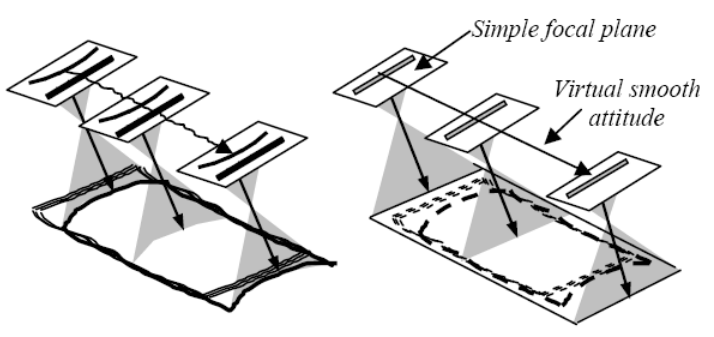

Figure 8: Perfect Sensor Geometry

This product is specially designed for the photogrammetic community, it can be accurately located by rational functions: in addition to Pleiades-HR physical model, metadata contains direct and inverse location models.

The production of this ideal linear array imagery is made from the raw image and its rigorous sensor model.

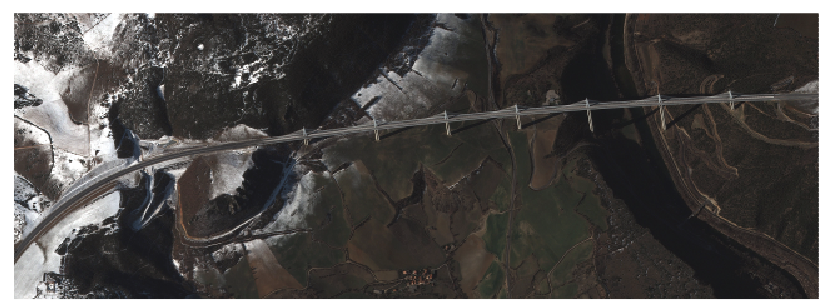

Figure 9: Pleiades-HR sensor image of Viaduc de Millau, (C) CNES Copyright 2012

The raw image is resampled into the Sensor Level geometry taking into account a DEM. The direct geolocation is made with an accurate Sensor Level geometric model

The Sensor Level product is delivered with two geometric models:

- a "rigorous sensor" model

- a rational function model.

Users can choose either the rigorous sensor model, or the rational function sensor model: results are very comparable.

\subsection{Ortho-image level}

The other set of products generated by the Pleiades-HR system are the ortho-images (and mosaic) products.

The Pleiades-HR ortho-image products are resampled into a cartographic projection and corrected from sensor and terrain distortions.

This product must be very precisely located to be used into geographic information systems (GIS). Product location is checked on an accurate DEM (Reference3D ${ }^{\mathrm{TM}}$, if available) with automatic GCPs (algorithm based on [2]). Users can also give their own DEM for orthorectification.

The final product volume is around 6 GBytes $(20 \mathrm{~km} * 20 \mathrm{~km})$. Pleiades-HR ortho-images can also be PAN-sharpened to obtain a $0.50 \mathrm{~m}$ GSD 4-band colour image.

\subsection{Mosaic products}

Mosaics products are larger size ortho-images automatically processed as a seamless patchwork of individual strips. This is made possible thanks to the high agility and the precise pointing capability of the platform and to a fully automatic mosaicing tool. This tool, named SIGMA, developed by CNES (BignaletCazalet, 2012) can automatically put each input image in a common geometry, homogenize the radiometry, and generate orthomosaics using stitching lines.

The Imagery files are composed of :

- 1 panchromatic mosaic of orthoimages file with $0.50 \mathrm{~m}$ GSD

- 1 multispectral mosaic of orthoimages with 2.00 m GSD

- Merged PA + XS, (blue, green, red, NIR) with PA GSD, enabling generation of a False Color (green, red, NIR) or Natural Color image (blue, green, red). 1 auxiliary data file

The final volume of a large mosaic can be up to 40 GBytes.

The tool has been at first validated on simulated data-strips from other sensors and intensively tested during commissioning phase with real adjacent acquired images. Several iterations with Image Quality experts and several re-processing were necessary for ground image parameters tuning according to each processing steps : spectral band registration, radiometric harmonization, stitching line computation, final product resampling.

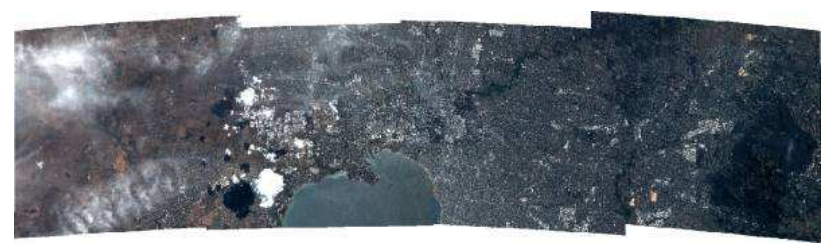

Figure 10. Pleiades-HR mosaic image of Melbourne, (C) CNES Copyright 2012

An operational requirement was to avoid any human intervention in the Pleiades Image Processing and it is a success. $60 * 60 \mathrm{~km}^{2}$ Mosaics have been produced in the main civilian center in less than 2 hours, with high level of parallelism of processes (more than 100 in operational conditions).

SIGMA is now a generic product, not only limited to images acquired in the same pass and already used for other optical missions.

\section{CONCLUSION}

A big amount of system products were necessary to perform the quality assessment and improve image processing and parameters during commissioning phase. All levels were necessary with an average of 60 product requests per day for calibration activities during the first 2 months. 


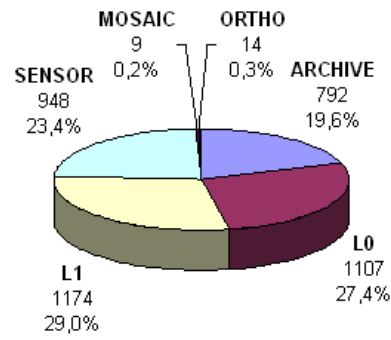

Figure 11- ICC Products requests distribution (Jan, Feb 2012)
Gleyzes, A. \& Al, 2012. Pleiades system architecture and main performances, ISPRS, Melbourne (Australia).

Kubik, K. \& Al, 2005. PLEIADES image quality: from users' needs to products definition, SPIE Europe, Brugges (Belgium).

Lebègue, L. \& Al, 2012. PLEIADES-HR image quality commissioning, ISPRS, Melbourne (Australia) .

PLEIADES ground segment and image processing software development was a hard, long but very interesting and exiting task. Many partners were involved in the process, from instrument design, algorithms specification to final tuning of parameters in operational centers. Teams have to manage delivery and maintenance of processing tools, as well as complete centers integration in many different possible configurations.

Images location, quality and performances have been continuously improved since the launch thanks to the ground processing systems.

The satellite acquires each day a maximum number of images, with imaging modes close to users' needs : targets, strips, stereo and tri-stereo, Corridor, one-pass mosaic or 'video mode'. A full range of products and quick access services are or will be soon available through Astrium Geo-Information Services portal for civilian needs.

The system allows to program, acquire, process and deliver the images in few hours, better than the required performances.

Now fully operational, the centers are preparing the operational qualification phase for the second satellite of the constellation.

\section{ACKNOWLEDGEMENTS}

The authors want to thank the whole team of partners who were involved in the Pleiades program development, specially image experts who designed innovative methods for continuously improving the performances of the images.

We want to acknowledge the industry partners for their high involvement until now for the development and integration of the processors into operational centers.

\section{REFERENCES}

Baillarin, S. \& Al, 2006. Remote Sensing Image Ground Segment Interoperability: PLEIADES-HR case study, Proceeding IGARSS, Denver (USA)

Baillarin, S. \& Al, 2009. Pleiades-HR system qualification. A Focus on ground processing and image products performances. A few months before launch. IGARSS, Cap Town (South Africa)

Bignalet-Cazalet, F. \& Al, 2012. Automatic and generic mosaïcing of Satellite images, ISPRS, Melbourne (Australia).

De Lussy, F. \& Al, 2006 . Pleiades-HR image system products and geometric accuracy, ISPRS, Hanover (Germany). 